\title{
Solidification Behavior of AZ31 Magnesium Alloys Using an Electromagnetic Vibration Technique
}

\author{
Mingjun LI, Takuya TAMURA and Kenji MIWA \\ National Institute of Advanced Industrial Science and Technology, Materials Research Institute for Sustainable Development, \\ Nagoya 463-8560 Japan. E-mail: li.mingjun@aist.go.jp
}

(Received on July 19, 2007; accepted on September 27, 2007)

\begin{abstract}
The commercial magnesium-based AZ31 alloy was solidified in a static magnetic field when an alternating electric current, perpendicular to the direction of the magnetic field, passes through the alloy. In this case, the periodical Lorentz force is generated, making the conductor vibrate centering on the initial equilibrium position. In this paper, we investigated the microstructure evolution of the alloy as a function of vibration frequency, magnetic flux density and electric current, respectively. The solidification behavior was discussed and the mechanism for the formation of the microstructure was proposed when considering the electrical properties of solid and liquid at high temperature. Because the electrical resistivity of liquid in the mushy zone is about twice that of the solid, this significant difference drives the solid to move faster than the liquid and thus generating a leading displacement for the solid over the liquid even within one vibration cycle. The uncoupled movement between the solid and liquid also gives rise to melt flow, which may be the reason to segment dendrites into fine particles. Meanwhile, the uncoupled movement makes it difficult to establish a steady state for solute redistribution during solidification and thus favoring equiaxed structures instead of dendrites. Considering these two factors, we examine the solidification behavior of the alloy separately when vibration frequency, magnetic flux density, and electric current are set as independent variables and the microstructure evolution as a function of these processing parameters can be well interpreted.
\end{abstract}

KEY WORDS: AZ31 magnesium alloy; microstructure formation; electromagnetic vibration; melt flow; solute redistribution.

\section{Introduction}

When an electric current passes through a conductor that is perpendicular to the direction of a magnetic field, the Lorentz force will be yielded and thus the conductor is forced to move in the magnetic field. If the direction of electric current varies cyclically, e.g., alternating current, the direction of the Lorentz force will be changed correspondingly with the same frequency as that of the alternating current according to the Fleming's left hand rule. In this case, the electromagnetic force drives the conductor to vibrate periodically, centering on the equilibrium position according to its initial boundary condition, which is termed electromagnetic vibration (EMV).

Based on this principle, Vives ${ }^{1,2)}$ employed the EMV technique to solidify a series of aluminum alloys and found that grain structures could be substantially refined when solidification was accomplished beyond a critical electromagnetic pressure peak. Efforts were also devoted to extend the EMV technique for continuous casting of $\mathrm{Al}$ alloys so as to achieve a refined structure in billet production. ${ }^{3)}$ With respect to the physical mechanism of the refined microstructure, Vives ${ }^{4)}$ mainly ascribed the phenomenon to cavitation that resulted in equiaxed grains.

In the past decade, Miwa and co-workers ${ }^{5-12)}$ further im- proved this technique to process alloys ${ }^{5-10)}$ and metals. ${ }^{11,12)}$ In addition to enhancing the glass forming ability of multiple component alloys upon the imposition of EMV, ${ }^{13)}$ they once more confirmed that crystallized microstructures could be greatly refined when the vibration frequency falls into an interval from $c a .200$ to $1000 \mathrm{~Hz}^{5-12)}$ The refinement was ascribed to the collapse of cavities and thus breaking the dendrites into debris, which, as they termed, was considered to be a micro-explosion process. ${ }^{5-12)}$

Cui and co-workers ${ }^{14,15)}$ are another research group who are engaged in EMV processing of metallic alloys for continuous billet casting. As the diameter of the billet reaches $270 \mathrm{~mm}$, they ${ }^{14,15)}$ employed a low frequency of up to $50 \mathrm{~Hz}$ to vibrate aluminum alloys, where the skin effect may not be so pronounced as it has an inverse proportion relation of the square root of frequency. Very recently, Wang et al. ${ }^{16)}$ employed the similar setup to study the grain refinement of AZ31 alloys under low-frequency electromagnetic casting.

As the $\mathrm{Mg}-3 \mathrm{wt} \% \mathrm{Al}-1 \mathrm{wt} \% \mathrm{Zn}(\mathrm{AZ31})$ alloy is typical for wrought processing, much work has been accomplished to throw light upon deformation mechanisms under various forging and extrusion conditions. However, microstructures obtained in a casting ingot prior to severe plastic deformation play an important role for subsequent processing as the alloy has a hexagonal closed packed (hcp) crystal lattice 
structure and thus having a fewer slip planes than those with a cubic structure. The anisotropic characteristic in deformation is rather pronounced for alloys with coarse structures and it is not favorable for a uniform deformation. However, the anisotropic feature can be greatly minimized by refined grains that randomly distribute throughout the entire volume, which is beneficial to have a uniform deformation during plastic processing. Moreover, a refined structure makes it possible to process at ambient temperature and thus reducing the production cost. StJohn et al. ${ }^{17)}$ summarized the influence of refined microstructures in $\mathrm{Mg}$ alloys on mechanical and chemical properties. Hence, a grain-refined structure of a solidified ingot is desirable from the viewpoint of industrial application even for the wrought AZ31 alloy.

The EMV technique has been demonstrated to be effective in producing grain-refined structures for alloys. In comparison with other approaches for the production of fine structures, e.g., rapid melt quenching and external incubation, a bulk ingot can be fabricated without any external additives during EMV processing. Furthermore, no attenuation in vibration intensity can be inferred throughout the entire volume of the sample provided that electric current can pass through the conductor uniformly, which forms a sharp contrast when compared with a steep amplitude decrease in ultrasonic and mechanical vibrations. Considering the aforementioned characteristics, we employed EMV to solidify AZ31 alloys under various conditions. Based on observed microstructures, we provide a new explanation for microstructure development when considering electrical properties of liquid and solid in the mushy zone.

\section{Experimental Procedure}

Commercial AZ31 alloy ingots were melted in an electric resistance furnace under the protection of a gas mixture of less than $2 \% \mathrm{SF}_{6}$ and balanced by $\mathrm{CO}_{2}$. As the liquidus temperature of the alloy is $905 \mathrm{~K}$, the alloy can be overheated to $955 \pm 10 \mathrm{~K}$, which has been verified to be conservative to avoid evaporating and maintain the alloy composition consistent with its initial ingredient. To make sufficient homogenization, the melt was held at the temperature for about 900 seconds and then poured into a preheated permanent mould to cast a rod.

A bar with a specification of $50 \mathrm{~mm}$ in length and $6 \mathrm{~mm}$ in diameter was made from the cast rod and then encapsulated into an $\mathrm{Al}_{2} \mathrm{O}_{3}$ tube, which was stabilized with carbon electrodes at two sides. A thermocouple with a sampling rate of 10 scans per second was embedded into an as-prepared hole in the $\mathrm{Al}_{2} \mathrm{O}_{3}$ tube to monitor the thermal profile during heating and cooling process. An arc-shaped carbon heater with a length of $40 \mathrm{~mm}$ was covered onto the tube to heat the sample. As the sample is $50 \mathrm{~mm}$ in length while the carbon heater is $40 \mathrm{~mm}$ in length, therefore, only the central part of the $\mathrm{Mg}$ rod can be melted during heating and thus leaving two extremes of the sample unmelted. These unmelted bulks contact with two graphite electrodes and thus no contamination can be involved from graphite electrodes as the melt does not contact them directly but grows from unmelted $\mathrm{Mg}$ parts. After the sample was melted, overheated, and then cooled to a desired temperature in a static magnetic field, EMV was yielded upon the imposition of the alternating current passing through the bar. When the sample was further cooled a well defined temperature, EMV was terminated by switching off the electric current, and simultaneously, the carbon heater was pulled up immediately. At the same time, cooling water with a flow rate of $6 \mathrm{~L} / \mathrm{min}$ was sprayed onto the tube to quench the sample so as to minimize a further coarsening of structures at the semisolid state. More details on the experimental procedure have been described elsewhere. ${ }^{11,12)}$

As there are three processing parameters involved in solidifying the alloy in the magnetic field, i.e., vibration frequency, $f$, magnetic flux density, $B_{0}$, and effective electric current, $J_{\mathrm{e}}\left(J=\sqrt{2} J_{\mathrm{e}} \sin 2 \pi f t\right)$, here we discussed the influence of these variables on solidification behavior, in particular the effect of solute redistribution and thermal effects on microstructure formation. In order to reveal the relation of vibration frequency as a function of microstructure development, eight different frequencies, i.e., $50 \mathrm{~Hz}, 100 \mathrm{~Hz}$, $250 \mathrm{~Hz}, 500 \mathrm{~Hz}, 750 \mathrm{~Hz}, 1000 \mathrm{~Hz}, 2000 \mathrm{~Hz}$, and $5000 \mathrm{~Hz}$, were selected while $J_{\mathrm{e}}$ and $B_{0}$ were kept as constants of $60 \mathrm{~A}$ and $10 \mathrm{~T}$. Similarly, $f$ and $J_{\mathrm{e}}$ were set as $500 \mathrm{~Hz}$ and $60 \mathrm{~A}$ when five different levels of magnetic flux density were selected, i.e., $1 \mathrm{~T}, 2.5 \mathrm{~T}, 5 \mathrm{~T}, 7.5 \mathrm{~T}$, and $10 \mathrm{~T}$ in terms of the effect of $B_{0}$ on structures. As far as the influence of electric current on microstructure is concerned, $f=500 \mathrm{~Hz}$ and $B_{0}=10 \mathrm{~T}$ were applied when the current levels were set as $10 \mathrm{~A}, 30 \mathrm{~A}, 60 \mathrm{~A}, 90 \mathrm{~A}$, and $120 \mathrm{~A}$, respectively.

The solidified samples were mounted, sectioned, ground, and then polished along traditional metallographic approaches for microstructure examination. As $\mathrm{Mg}$ alloys are active to react with moisture and easy to be oxidized in air, special attention was paid throughout the entire preparation process. After the sample was well polished, an etchant solution with acetic acid $(10 \mathrm{~mL})$, picric acid $(4.2 \mathrm{~g})$, distilled water $(10 \mathrm{~mL})$, and ethanol $(70 \mathrm{~mL})$ was used to reveal grain structures. The microstructures were observed under an optical microscope and the average grain size, $d$, was measured using an Image Pro- ${ }^{\circledR}$ software package. The average diameter of a grain was measured using the centroidal method, i.e., the mean length of the grain was obtained at two degree intervals when passing through the grain's centroid. To ensure a reliable result, microstructures in four areas for each sample were randomly taken for measurement.

In order to reveal the crystallographic relation of microstructures after solidification, the electron backscatter diffraction (EBSD) system from EDAX-TSL was employed to characterize the orientation distribution of grains. To generate clear Kikuchi patterns for indexing, a perfect surface free from deformation or contamination was a must for the EBSD analysis. Therefore, further fine polishing was carried out using $0.25 \mu \mathrm{m}$ diamond slurry and then the sample was etched in an ion-beam shower system (EIS200ER) to remove the deformed surface layer. Once ion beam etching was completed, the sample should be set immediately into the chamber of the scanning electron microscope (SEM) from JEOL (JSM-5910) to avoid oxidizing and reacting with moisture during expanded exposure in air. In terms of data acquisition, one-step scanning generated a single Kikuchi pattern that corresponded to an indexing 

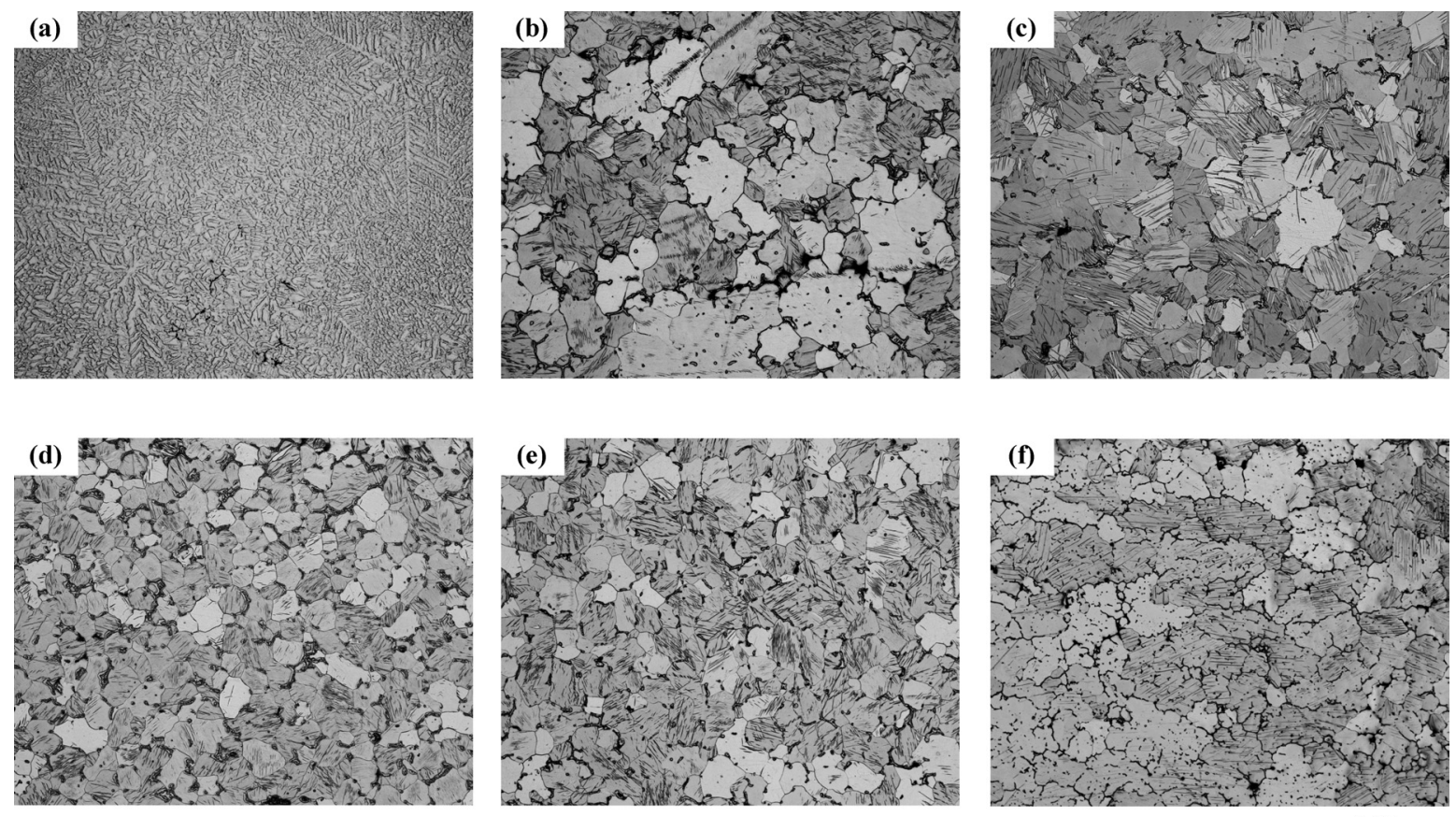

$\underline{200 \mu \mathrm{m}}$

Fig. 1. The optical microstructures of AZ31 alloys solidified (a) under the as-cast condition in a preheated metallic mould and at (b) $f=50 \mathrm{~Hz}$, (c) $f=100 \mathrm{~Hz}$, (d) $f=500 \mathrm{~Hz}$, (e) $f=1000 \mathrm{~Hz}$, and (f) $f=5000 \mathrm{~Hz}$ under the vibration condition when magnetic flux density and electric current are set as $B_{0}=10 \mathrm{~T}$ and $J_{\mathrm{e}}=60 \mathrm{~A}$, respectively.

pixel, which was represented as one pixel in the EBSD map in the subsequent data analysis. The raw dataset was subjected to a standard grain dilatation clean up (grain dilatation $5^{\circ}$ and minimum grain size $2 \mu \mathrm{m}$ ). The EBSD color map was displayed using the index of image quality and inverse pole figure to decode the pattern.

\section{Experimental Results}

\subsection{Microstructure Evolution versus Vibration Fre- quency}

Figure 1 depicts five microstructures of the alloy solidified at various vibration frequencies under the condition of $B_{0}=10 \mathrm{~T}$ and $J_{\mathrm{e}}=60 \mathrm{~A}$. For comparison, a microstructure observed at the normal cast condition is also included, exhibiting coarse dendrites with well developed trunks and arms, as shown in Fig. 1(a). It can be seen that at a low vibration frequency, e.g., $f=50 \mathrm{~Hz}$, rosette-like structures are yielded with some fine equiaxed grains distributing at the interval region, as indicated in Fig. 1(b). With the increase of vibration frequency, the solidified structure becomes fine and within the frequency interval from about $f=500 \mathrm{~Hz}$ to $f=2000 \mathrm{~Hz}$, a unique structure with equiaxed grains can be observed. A further increase of vibration frequency to $5000 \mathrm{~Hz}$ results in an increase in grain size, exhibiting a similar coarse microstructure with that observed at $f=50 \mathrm{~Hz}$.

To have a quantitative characterization of these structures, we measured the average grain size as a function of $f$, as summarized in Fig. 2, in which the solid squares show the measured data. Two short bars at the extreme of a straight line are the upper and lower average grain sizes at the frequency, where the upper one is obtained by ranking all grains measured from large to small and then determined at grain number fraction of $20 \%$ while the lower is

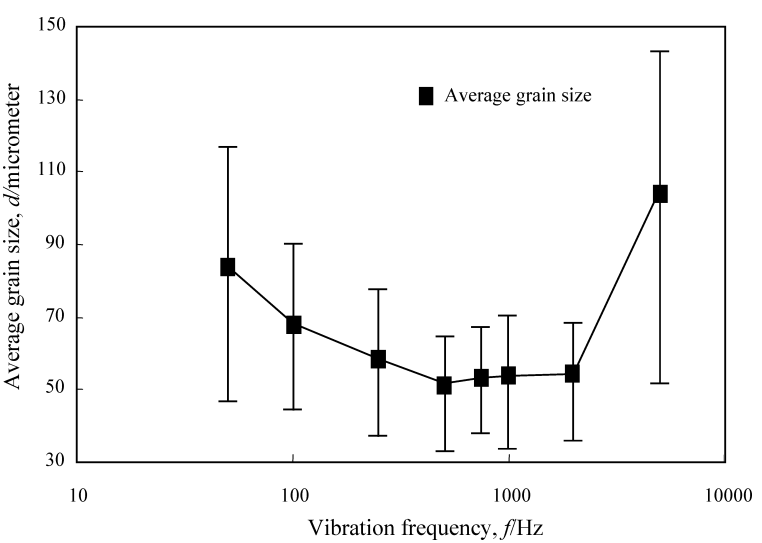

Fig. 2. The measured grain size of AZ31 alloys as a function of vibration frequency. The solid squares indicate the average grain size. When all grains are sequenced from large to small, the diameters of grains at of $20 \%$ and $80 \%$ of all measured grains are marked by an upper and lower short bars at the given frequency. One can readily tell that the length of the line indicates the scattering scope of grain distribution.

achieved at the fraction of $80 \%$. Therefore, the length of the straight line indicates the scattering level of grain size distribution; the longer the line is, the larger the scale for the grain sizes can be inferred. Obviously, the grain size covers a large scale at both low and high frequencies while it falls into a narrow interval when structures exhibit round and equiaxed grains at the frequency range from $\mathrm{ca} .500 \mathrm{~Hz}$ to less than $2000 \mathrm{~Hz}$. This is consistent with the microstructure evolution versus vibration frequency, where a hybrid structure with coarse dendrites and fine grains can be found at low and high vibration frequencies while a unique structure with fine equiaxed grains can be identified at the medium frequency band. 

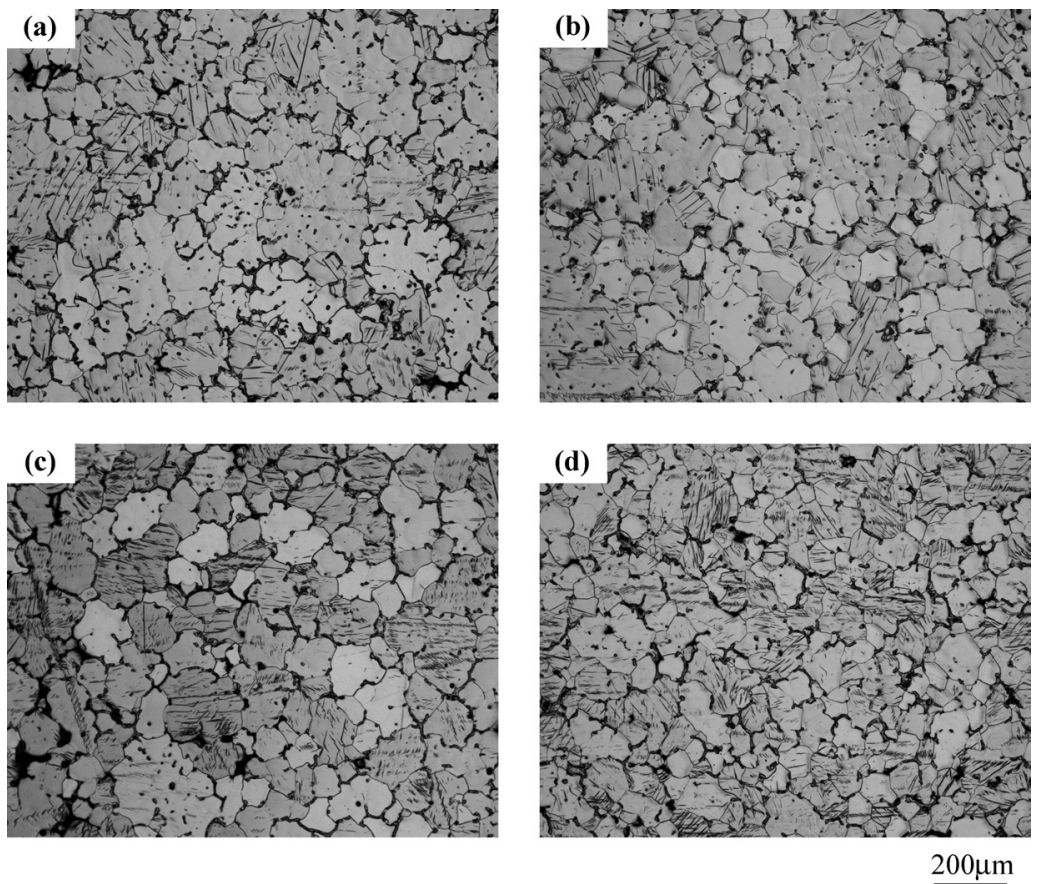

Fig. 3. The optical microstructures of AZ31 alloys solidified at different magnetic flux densities of (a) $B_{0}=1 \mathrm{~T}$, (b) $B_{0}=2.5 \mathrm{~T}$, (c) $B_{0}=5 \mathrm{~T}$, and (d) $B_{0}=7.5 \mathrm{~T}$ when vibration frequency and electric current are set as $f=500 \mathrm{~Hz}$ and $J_{\mathrm{e}}=60 \mathrm{~A}$, respectively.

\subsection{Microstructure Evolution versus Magnetic Flux Density}

As the microstructure can be refined at processing parameter of $f=500 \mathrm{~Hz}$ and $J_{\mathrm{e}}=60 \mathrm{~A}$, we set this frequency and electric current when revealing the relation of microstructure evolution as a function of $B_{0}$. Figure 3 depicts four microstructures of the alloy solidified at various magnetic flux densities. One can see that at a low $B_{0}$ of $1 \mathrm{~T}$, the primary structure is coarse and the dendritic skeleton can be discerned in Fig. 3(a). When $B_{0}$ is increased to $2.5 \mathrm{~T}$, the number of coarse grains decreases and in the meanwhile, the more equiaxed grains can be found in boundary areas. With a further increase of $B_{0}$ to $5 \mathrm{~T}$, the grain structure becomes much finer and the number fraction of equiaxed grains accordingly increases, as shown in Fig. 3(c). When $B_{0}$ is increased from 7.5 to $10 \mathrm{~T}$, structures are uniform and only equiaxed grains can be observed in Fig. 3(d) and Fig. 1(d).

Similar to the grain size measurement in Sec. 3.1, we adopt the same approach to characterize the grain structure distribution versus magnetic flux density. Figure 4 indicates the measured data of average grain size as a function of $B_{0}$, as highlighted by the solid squares. One can see that there is a sharp decrease in average grain size from about $110 \mu \mathrm{m}$ at $B_{0}=1 \mathrm{~T}$ to $c a .75 \mu \mathrm{m}$ at $B_{0}=2.5 \mathrm{~T}$. A further increase of $B_{0}$ from 2.5 to $10 \mathrm{~T}$ only leads to a deduction from $75 \mu \mathrm{m}$ to about $50 \mu \mathrm{m}$. The same definitions for the distribution of upper and lower average grains are applied as that presented in Sec. 3.1 to reveal the scattering degree of structures, as depicted by the length of the straight line. It is obvious that with the increase of $B_{0}$, the scattering interval decreases from a wide range at $B_{0}=1 \mathrm{~T}$ to a narrow range at $B_{0}=7.5 \mathrm{~T}$ and keeps almost a constant from $B_{0}=7.5 \mathrm{~T}$ to $B_{0}=10 \mathrm{~T}$ with a rather small gap, indicating that a uniform structure prevails at this magnetic field level. This variation

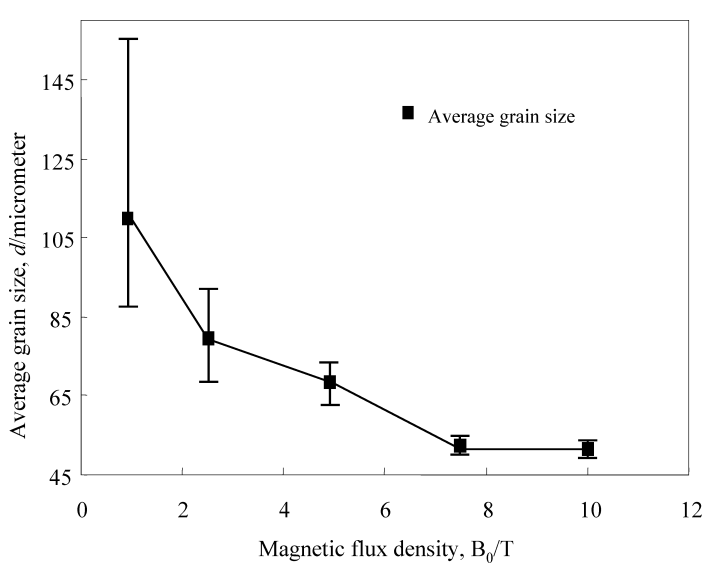

Fig. 4. The measured grain size of AZ31 alloys as a function of magnetic flux density. The solid squares indicate the average grains size under different levels of $B_{0}$. With the same definition as that in Fig. 2, the upper and lower short bars are included to indicate the grain distribution.

agrees well with the microstructure observation where there is a hybrid structure when $B_{0}$ is up to $7.5 \mathrm{~T}$ and there is a unique structure with equiaxed grains when $B_{0}$ is increased from 7.5 to 10 T, as illustrated in Fig. 3(d) and Fig. 1(d).

\subsection{Microstructure Evolution versus Electric Current}

As the aforementioned investigation reveals, the microstructure could be refined from $f=500 \mathrm{~Hz}$ at $B_{0}=10 \mathrm{~T}$, we set this vibration frequency and magnetic field in order to find proper electric current. Figure 5 shows four microstructures observed at different levels of electric current. Similar to that identified at a low $B_{0}$, the structure is rather coarse when $J_{\mathrm{e}}=10 \mathrm{~A}$. With the increase of $J_{\mathrm{e}}$, more equiaxed grains are yielded. To avoid repeating, the microstructure obtained at $60 \mathrm{~A}$ is omitted here as it is similar 

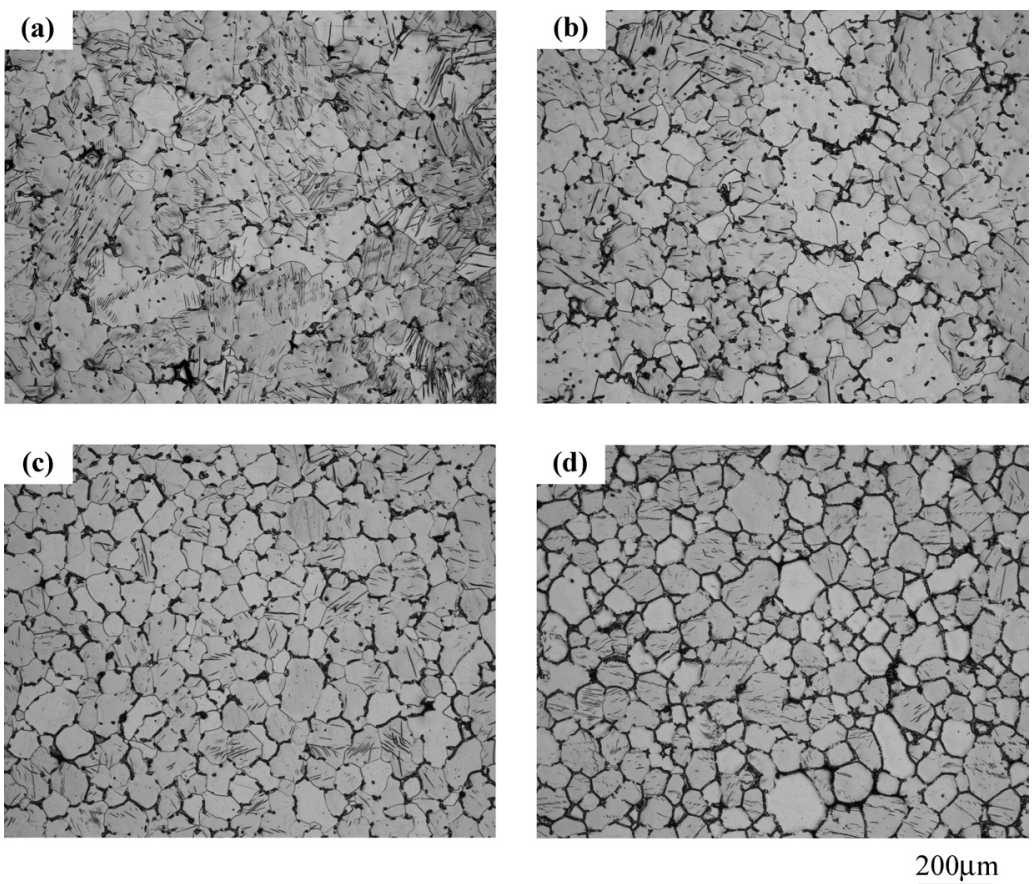

Fig. 5. The optical microstructures of AZ31 alloys solidified at different levels of electric current of (a) $J_{\mathrm{e}}=10 \mathrm{~A}$, (b) $J_{\mathrm{e}}=30 \mathrm{~A}$, (c) $J_{\mathrm{e}}=90 \mathrm{~A}$, and (d) $J_{\mathrm{e}}=120 \mathrm{~A}$ when vibration frequency and magnetic flux density are set as $f=500 \mathrm{~Hz}$ and $B_{0}=10 \mathrm{~T}$, respectively.

to Fig. 1(d) observed under the same processing condition. When $J_{\mathrm{e}}$ is further increased from $J_{\mathrm{e}}=90 \mathrm{~A}$ to $J_{\mathrm{e}}=120 \mathrm{~A}$, unique structures with equiaxed grains can be yielded, which exhibit a similar morphological feature with that observed at $J_{\mathrm{e}}=60 \mathrm{~A}$.

Following the same procedure for grain size measurement, we also make a quantitative analysis to correlate the grain size as a function of $J_{\mathrm{e}}$, as illustrated in Fig. 6. One can see that there is a substantial decrease in average grain size from $J_{\mathrm{e}}=10 \mathrm{~A}$ to $J_{\mathrm{e}}=60$ A. No pronounced decrease can be inferred from $J_{\mathrm{e}}=60 \mathrm{~A}$ to $J_{\mathrm{e}}=90 \mathrm{~A}$ where it reaches the minimum of about $48 \mu \mathrm{m}$. On the contrary to the monotonic decrease in grain size with the increase of $B_{0}$ in Fig. 4 , a further increase of electric current to $J_{\mathrm{e}}=120 \mathrm{~A}$ results in an slight increase to about $50 \mu \mathrm{m}$. But for the quantitative measure based on the software, it should be difficult to tell this minute difference only by observing Figs. 5(c) and $5(\mathrm{~d})$. With regard to the grain structure distribution, it can be seen that at a low electric current, i.e., $J_{\mathrm{e}}=10 \mathrm{~A}$, the structure covers a wide length scale in diameter, consistent with the microstructure observation. When a unique structure with round grains is yielded, the gap between the upper and lower bars reaches the minimum at about $J_{\mathrm{e}}=60 \mathrm{~A}$ and a further increase of electric current leads to an increase in the line length. Here it should be noted that experimental failure always occurs when $J_{\mathrm{e}}$ is increased higher than $120 \mathrm{~A}$; this is why no data are available beyond this critical value.

\section{Discussions}

The physical mechanism for structure refinement in EMV process has been controversial. ${ }^{4}$ Vives first proposed that cavitation take place in melt during vibration, which resulted in the formation of refined homogeneous microstruc-

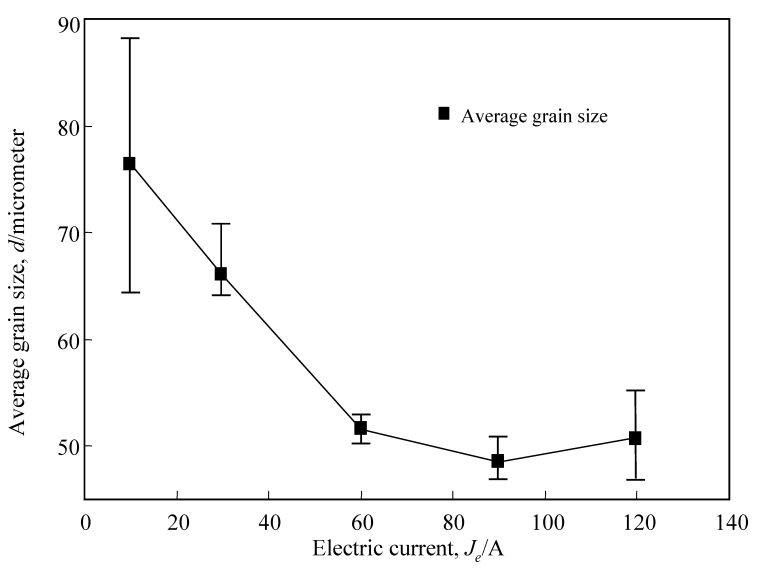

Fig. 6. The measured grain size in AZ31 alloys as a function of electric current. The solid squares indicate the average grain size under different levels of $J_{\mathrm{e}}$. With the same definition as that in Fig. 2, the upper and lower short bars are included to indicate the grains distribution.

tures. ${ }^{1,2)}$ Similarly, Miwa and co-workers ${ }^{5-12)}$ suggested that the collapse of bubbles in melt during vibration produce strong shock wave that makes dendrites break into fragments and thus refining microstructures, which they termed as a micro-explosion process. ${ }^{5-12)}$ Note that Vives ${ }^{4)}$ also mentioned that severe agitation in melt during vibration may transport newly formed nuclei to distribute throughout the solidifying pool. One major question suspending for the cavitation mechanism is that refinement always can be produced in an interval from several hundred up to $2000 \mathrm{~Hz}$, which is about one to two orders of magnitude lower than the well-defined ultrasonic frequency band. If cavitation could really be responsible for the fragmentation, grain refined microstructure should be readily produced when vibration is operated near or at the ultrasonic frequency range 
where cavitation has been confirmed to readily occur. ${ }^{18,19)}$ Unfortunately, complete dendrites with well developed trunks were obtained in Al-Si alloys when vibration was completed at $10 \mathrm{kHz},{ }^{6}$ a frequency near to the ultrasonic frequency band; this result contradicts with the cavitation mechanism and thus making the applicability of the mechanism ambiguous during EMV processing.

When EMV is imposed upon a conductor, it oscillates while centering on its initial equilibrium position. For an alloy with a freezing interval where the solid and liquid coexist in a mushy zone, both the solid and the liquid vibrate around their equilibrium positions. If the solid and the liquid vibrate synchronically, i.e., with the same amplitude at the same displacement and velocity, no interactions can be inferred to occur between the solid and liquid. In this case, the boundary condition of solute redistribution should not be altered and thus the growth of dendrites should be favored. On the contrary to this assumption is that refinement is more effective when vibration is operated at the mushy zone, indicating that EMV process gives rise to interactions between the solid and liquid. Here we shall consider the origin of interactions that lead to the formation of equiaxed grains.

The Lorentz force to which a conductor in a magnetic field is subjected drives the conductor to move, which obeys Newton's second law of motion. Therefore, the acceleration can be obtained as, $a=L \cdot B_{0} J_{\mathrm{e}} / m$ when assuming that vibration takes place in free space with $m$ being the mass and $L$ the length of the conductor. From acceleration, one can make preliminary calculations of main peak parameter values of velocity, $v=\sqrt{2} L \cdot B_{0} J_{\mathrm{e}} /(2 \pi f \cdot m)$ and displacement, $s=\sqrt{2} L \cdot B_{0} J_{\mathrm{e}} /\left(4 \pi^{2} f^{2} \cdot m\right)$. For a specific volume of solid and liquid in the mushy zone at high temperature, the most striking feature lies in the fact that a significant difference arises in electrical resistivity between the solid and the liquid. Good examples to clarify this issue comes from electrical properties of pure metals of $\mathrm{Mg}, \mathrm{Al}$, and $\mathrm{Cu}$ at their melting point, which are $274 \mathrm{n} \Omega \cdot \mathrm{m},{ }^{20)} 242 \mathrm{n} \Omega \cdot \mathrm{m},{ }^{21)}$ and $215 \mathrm{n} \Omega \cdot \mathrm{m}^{22}$ in their liquid states, respectively, while they are $154 \mathrm{n} \Omega \cdot \mathrm{m},{ }^{20)} 110 \mathrm{n} \Omega \cdot \mathrm{m}^{21)}$ and $110 \mathrm{n} \Omega \cdot \mathrm{m}^{22)}$ in their solid states at the same temperature. Although there are no accurate data for the present AZ31 alloy, the electrical resistivity can be approximately evaluated since the electrical resistivity is more or less proportional to the amount of solute added. ${ }^{20)}$ Hence, it is reasonable to deduce that the electrical resistivity of the liquid is about twice of that the solid as $\mathrm{Mg}$ and $\mathrm{Al}$ are two major components. When the Ohm's law applies for these two phases in the mushy zone, the remarkable difference in electrical resistivity makes electric current in solid as high as twice that in liquid. Therefore, one can readily tell that the solid moves with a larger acceleration, a higher velocity, and thus covering a larger displacement in comparison with that of the liquid, i.e., an uncoupled movement can occur and thus there are interactions between the solid and liquid.

The consequences induced by such a kind of uncoupled movement can be delineated from three different aspects. First, the uncoupled movement between solid and liquid can induce melt flow or convection in the mushy zone. A general belief is that melt flow can promote grain refinement by a dendrite fragmentation mechanism, although there is not yet agreement on what that basic mechanism is. ${ }^{23)}$ Hellawell et al. $^{24)}$ summarized the process as following five steps: (1) local remelting of arms at necks, (2) transportation of segments, (3) survival against remelting, (4) further growth, and (5) formation of equiaxed grains to impede dendrite growth. As vigorous convection takes place during EMV processing, this fragmentation process may operate to multiple grains as well and thus refined microstructures can be yielded.

Second, melt flow within the mushy zone can rotate or transport segmented crystals and thus destroying the crystallographic orientation of grains from one dendrite, which, otherwise, should have the same orientation if they originate from a single dendrite. A good example to demonstrate the influence of melt flow on these first two aspects comes from a comparison of microtextures of $\mathrm{Ni}_{99} \mathrm{~B}_{1}$ alloys solidified on an electromagnetic levitator (EML) and an electrostatic levitator (ESL), ${ }^{25)}$ where melt flow induced by electromagnetic force occurs in a sample processed on the EML while a quiescent state can be inferred in the melt levitated on the ESL when a laser beam is utilized to heat the sample in which mechanical disturbance induced by electromagnetic stirring can be avoided. At low and medium undercoolings, equiaxed grains can be yielded in alloys solidified on the EML with a random distribution in crystallographic orientations while complete dendrite skeletons can be identified in spheres processed on the ESL in which fine grains in among dendrite arm areas share the same crystallographic orientation as that of the dendrite. ${ }^{25)}$ Figure 7(a) shows a colorful EBSD map of the AZ31 alloy solidified after vibration at $f=100 \mathrm{~Hz}$ where the scanning step during data acquisition is set as $4 \mu \mathrm{m}$. Correspondingly, the pole figure of the EBSD is depicted, as shown in Fig. 7(b), from which one can see that the distribution of overall structure is random. When the misorientation relation of neighboring boundaries is revealed in Fig. 7(c), it shows that more than $40 \%$ of boundaries have a small misorientation angle less than 10 degree. This can be further confirmed from the grains color where several neighboring grains share a similar color. Note that using the EBSD technique, it cannot be applied to characterize dislocations, ${ }^{26}$ ) while it can detect the low-angle interfaces induced by dislocations, which exhibit small misorientation angles. For the AZ31 alloy, some low-angle interfaces may be included.

Third, the mechanical interaction, which can be expressed as shear stress induced by the uncoupled movement between solid and liquid, can produce some deformation on the primary solid. As AZ31 requires a rather low critical resolved shear stress of less than $3 \mathrm{MPa}^{27)}$ in yielding the $\{10 \overline{1} 2\}\langle 10 \overline{1} 1\rangle$ twins, this type of twin is often observed whereas other potential twinning systems, e.g., $\{10 \overline{1} 1\}\langle 11 \overline{2} 0\rangle,\{10 \overline{1} 2\}\langle 11 \overline{2} 0\rangle$ or $\{10 \overline{1} 0\}\langle 11 \overline{2} 0\rangle$ require a larger equivalent stress. In the $\{10 \overline{1} 2\}\langle 10 \overline{1} 1\rangle$ system, the twinning leads to a misorientation of $86.3^{\circ}$ at twinned crystal planes. ${ }^{28,29)}$ This can be confirmed in Fig. 7(c), where the number fraction near the misorientation of $90^{\circ}$ is as high as about $20 \%$, ranking the second after the low misorientation angle. Some grains with twins are highlighted in dashed circles in Fig. 7(a). One can see that in comparison with the morphological appearance of annealing twins in Ni-based cubic alloys where there is always a clear twin- 

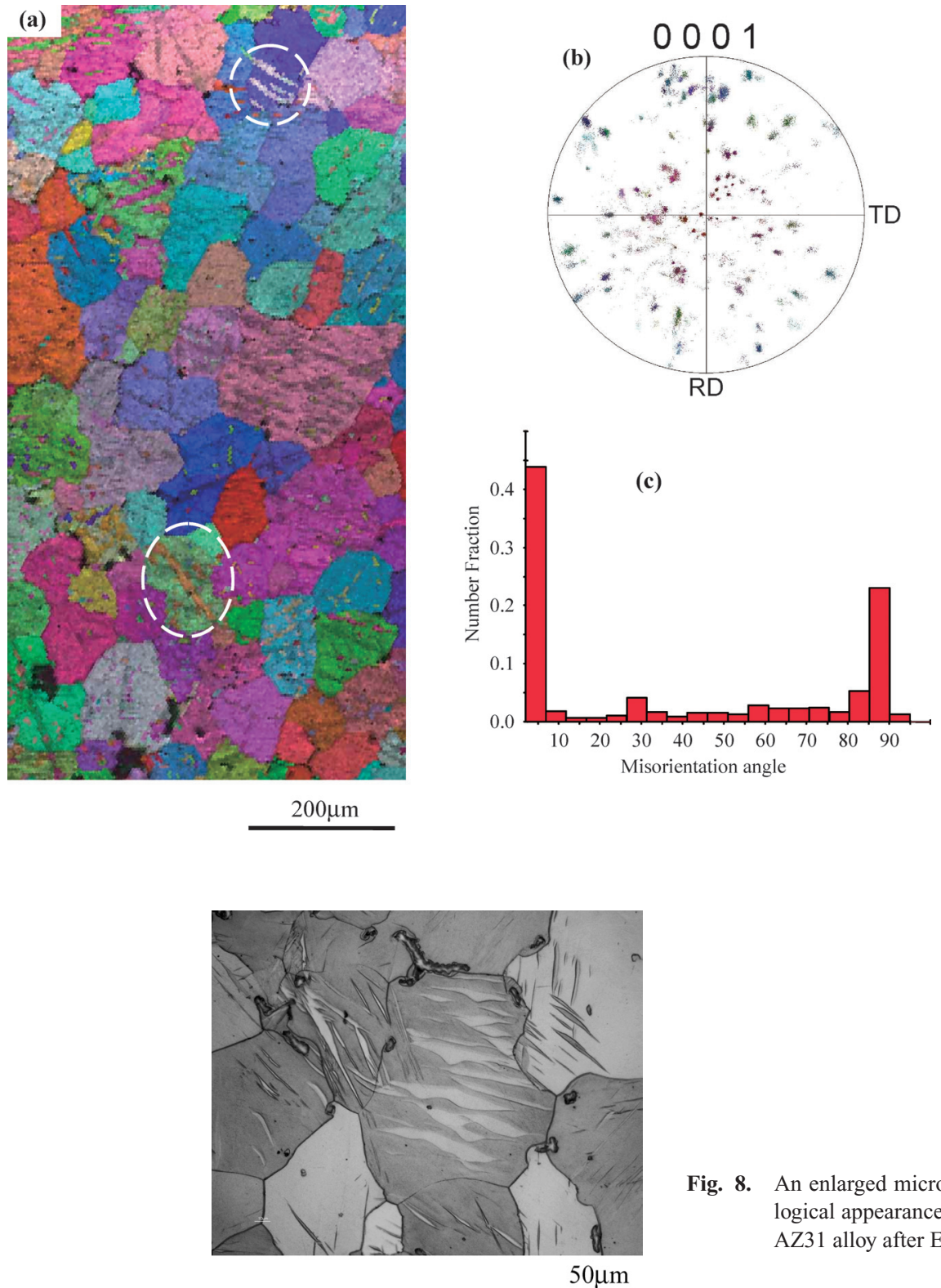

Fig. 7. (a) An EBSD pattern of the AZ31 alloy, which is decoded by the image quality and inverse pole figure, (b) the pole figure, and (c) the histogram showing the number fraction versus misorientation angle on the basis of the EBSD.
Fig. 8. An enlarged microstructure showing the morphological appearance and width scale of twins in the AZ31 alloy after EMV processing. (a)

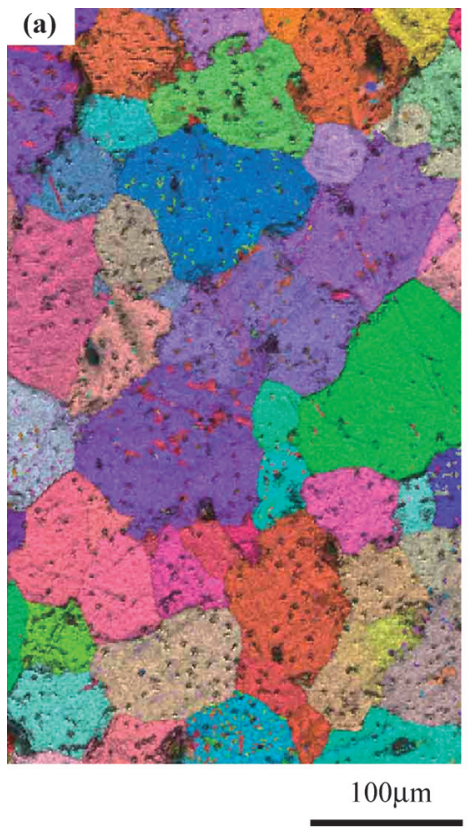

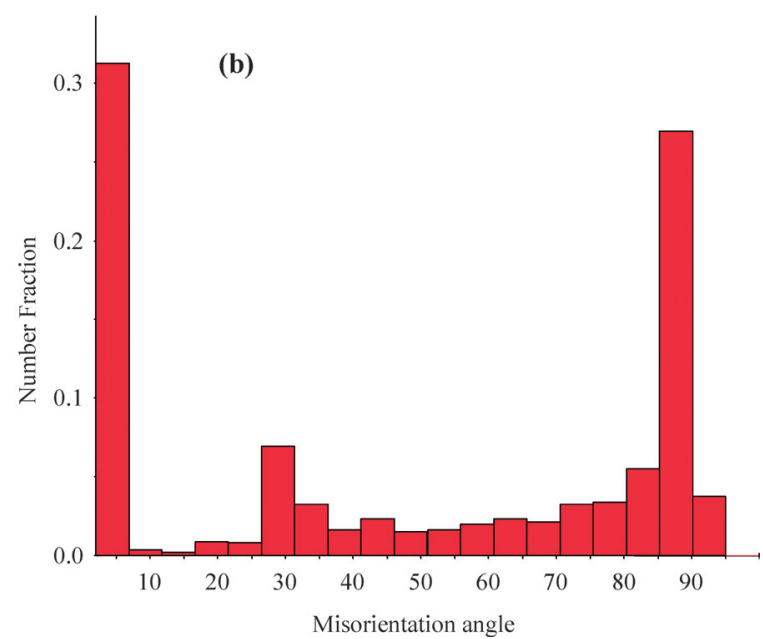

Fig. 9. (a) An EBSD pattern of the AZ31 alloy with a fine scanning step of $1.5 \mu \mathrm{m}$ and (b) a histogram of the number fraction versus misorientation angle according to the EBSD map, showing an increased number fraction near $90^{\circ}$ due to fine scanning. 
ning boundary splitting sub-grains into two parts, ${ }^{25)}$ the twinned bands in the present AZ31 alloy exhibit a cellular or lath morphology, covering from several micrometers to tens of micrometers in width, as depicted in Fig. 8, which shows an enlarged microstructure. This morphological feature makes the result of misorientation versus number fraction in EBSD analysis dependent on the scanning conditions. When the scanning step is larger than the width of a twin, the twin may not be revealed in the EBSD map while only the crystallographic characteristic of surrounding matrix is detected. Figure 9(a) shows an EBSD map that is obtained at a fine scanning step of $1.5 \mu \mathrm{m}$. From Fig. 9(b), one can see that the number fraction near $90^{\circ}$ reaches almost the same as that at small misorientation angles in the histogram of number fraction versus misorientation angle. This should be attributed to the reduced scanning step that enables more twins to be detected.

Here it should be supplemented that in contrast to a large number of twins in the AZ31 alloy, one never observes any twins in rounded grains by other semi-solid processing techniques, in which shear stresses are involved and a relative motion between the solid and liquid can usually be inferred in slurries. The primary reason for this pronounced difference may lie in the fact that the critical stress for twin formation in the AZ31 alloy is rather low. ${ }^{27)}$ For some other alloys, e.g. Al-7\% $\mathrm{Si}$ alloys, ${ }^{9)}$ no twins were revealed during EMV processing even when a larger vibration force $\left(J_{\mathrm{e}}=120 \mathrm{~A}\right.$ and $\left.B_{0}=10 \mathrm{~T}\right)$ was imposed; this twin-free feature is similar to that observed in forced convection for $\mathrm{Al}-6.5 \% \mathrm{Si}$ alloys ${ }^{23}$ because of the very high stacking fault energy of aluminum. ${ }^{30)}$ Employing the same processing parameters as for AZ31 alloys, we ${ }^{31}$ ) have actually solidified AZ91D alloys (with a nominal composition of $\mathrm{Mg}-$ $9 w t \% \mathrm{Al}-1 \mathrm{wt} \% \mathrm{Zn}$ for die casting processing) using the present EMV setup; however, no twins could be identified as it may be a reluctant twin-forming alloy.

The difference in electrical resistivity between solid and liquid for the present AZ31 alloy at high temperature is a constant, indicating that an uncoupled movement between two phases always exists once EMV is imposed. However, refinement induced by such a relative movement cannot be identified throughout the entire frequency range but only confined within a narrow interval, i.e., from ca. $500 \mathrm{~Hz}$ up to $2000 \mathrm{~Hz}$. In order to explain such a frequency-dependent refinement, $\mathrm{we}^{32)}$ have recently calculated the distance covered by the leading solid and the equivalent solute redistribution boundary layer when assuming $2 J_{\text {e(liquid) }} \approx J_{\text {e(solid) }}$. A further detailed analysis reveals that refinement becomes effective only at a certain frequency range where the leading distance covered by the mobile solid is as large as the thickness of the solute boundary layer. In this case, the solute rejected by the crystallizing solid cannot be piled up ahead of the solid/liquid interface in a steady-state manner. As is known, constitutional undercooling is mainly responsible for the destabilization of a planar interface to form high order branches of dendrites. In order to achieve constitutional undercooling, the pileup or depletion of solute at the solid/liquid interface is a must in generating constitutional undercooling even when the temperature gradient is not so steep. In the present case, since the solute cannot be piled up steadily in front of the solid/liquid interface, no constitutional undercooling can be inferred to occur; therefore, it is hard to destabilize a growing crystal to form high order of dendritic branches. It is worth noting that the distance covered by the mobile solid decreases substantially per a square relation with vibration frequency. This makes the relative displacement become rather short when vibration frequency is increased to a high level. In this case, the vibration amplitude of solid is so small that it is confined within the operating region of solute boundary layer, i.e., the vibration cannot alter the solute redistribution condition and thus a coarse structure can be produced, similar to that during normal casting.

Here it should be noted that we only discussed a simple case with one dendrite immersing into liquid, where the typical length scale is the radius of the dendrite tip in the mushy zone. In practice, a bundle of dendrites always grow; however, the present analysis still stands for this growth behavior as the primary trunk spacing is much larger than the radius of the tip. When the dendrite tip becomes large to retrograde a cellular or planar interface, the fragmentation of the trunks becomes difficult as the volume fraction of liquid is quite low. This may be the reason that the minimum average grain size of pure metals after vibration is always larger than that of alloys, e.g., for pure $\mathrm{Mg}^{11)}$ and $\mathrm{Al}^{12)}$ metals, it is usually about $200 \mu \mathrm{m}$ whereas for alloys, it is always around $50 \mu \mathrm{m}^{1)}$ or smaller. ${ }^{9)}$ In the meanwhile, it should be noted that although the calculated interface undercooling may be rather low covering a narrow range of less than about $2 \mathrm{~K},{ }^{32)}$ the actual interface undercooling of each individual growing crystal may differ from tip to tip for a bundle of dendrites crystallizing during cooling, which will make the thickness of solute boundary layer differ from tip to tip. This distribution enables some dendrites to be readily segmented into fine particles while other dendrites are probably too robust to fragment into fine grains but exhibiting coarse structures. This may be the reason that one can frequently find a hybrid structure when vibration amplitude is in a marginal level but a unique structure with round grains when vibration is operated at the region where the solute boundary for all dendrites cannot be maintained any more.

As far as the influence of $B_{0}$ on the microstructure formation is concerned, one can tell that the Lorentz force to which the conductor is subjected is proportional to $B_{0}$, resulting in a linear variation in acceleration, relative velocity and relative displacement. This indicates that the larger the $B_{0}$ is, the higher the relative acceleration and relative velocity should be obtained, and thus yielding a longer displacement within a specific vibration interval. Meanwhile, with the increase of $B_{0}$, the interactive shear force between the solid and liquid increases and thus making the fluid flow more severe, which can be of great help in promoting fragmentation process. Considering these two effects, it is, therefore, easy to understand the microstructure evolution of AZ31 alloys as a function of magnetic flux intensity; coarse structures are formed at low $B_{0}$ as the relative distance covered by the leading solid may be so small that the vibration amplitude is always within the operating region, while the increased driving force for fragmentation makes dendrites break up into fine particles at high $B_{0}$ and thus yielding refined structures, as indicated in Fig. 4. 
As we mentioned, vibration can result in refinement; meanwhile, it can produce deformation twins in the alloy. Similar to refinement in driving force, the formation of deformation twins requires driving force as well. At a given electric current, electromagnetic force increases linearly when $B_{0}$ is increased, which can activate more and more crystal planes to slip to form deformation twins. From Fig. 3 , one can see that the number of twinned grains increases with the increase of $B_{0}$ whereas no twins are produced when the alloy is cast in a permanent mould since no driving force arises for deformation, as shown in Fig. 1(a). Here it is worth noting that twins cannot be found in every grain. This should be attributed to the fact that the orientation of individual grain may be different due to melt stirring whereas the vibration force has a strong directionality. Slipping can only be activated to take place along those planes that require a low critical shear stress and thus leaving some grains free from twinning because of their unfavorable orientation alignment.

In terms of influence of $J_{\mathrm{e}}$ on microstructure evolution, one can see that, at a constant $B_{0}$, vibration force is proportional to electric current, which is similar to the variation of $B_{0}$ provided that the electric current is specified. As a linear relation can be found, the relative velocity and displacement increase proportionally, making the driving force for fragmentation increase regarding to destabilizing the solute boundary layer and promoting the melt flow, and thus yielding a decrease in grain size with the increase of $J_{\mathrm{e}}$, as shown in Fig. 6. The mechanism for the microstructure development is similar to that in the previous discussion with respect to the effect of magnetic field. Therefore, to avoid repeating, we shall omit an expanded discussion on the issue but concentrate on the differences when magnetic field and electric current are employed separately.

The most striking feature is that different amounts of joule heat are produced at different levels of $J_{\mathrm{e}}$ at $B_{0}=10 \mathrm{~T}$ while joule heat for each sample maintains almost a constant at various densities of $B_{0}$ at $J_{\mathrm{e}}=60 \mathrm{~A}$. When $J_{\mathrm{e}}$ is less than $60 \mathrm{~A}$, joule heat is small and its influence can be negligible because no difference arises in slope of the cooling curves. However, when $J_{\mathrm{e}}$ reaches $90 \mathrm{~A}$, a detectable difference appears in that the cooling rate becomes slow in comparison with that at $J_{\mathrm{e}}=60 \mathrm{~A}$. When $J_{\mathrm{e}}$ is further increased to $J_{\mathrm{e}}=120 \mathrm{~A}$, cooling becomes rather slow and total cooling process covers a quite long period from 953 to $853 \mathrm{~K}$. In this case, the influence of joule heat on the microstructure cannot be neglected any more. When electric current increases from $J_{\mathrm{e}}=90 \mathrm{~A}$ to $J_{\mathrm{e}}=120 \mathrm{~A}$, no decrease in grain size, on the contrary, a slight increase in grain size can be found which should be ascribed to an extended duration in the semisolid state, where structure coarsening takes place in the mushy zone. As the electric resistivity of solid differs from that of liquid, making the production of joule heat different between the solid and liquid, this non-uniformity feature results in a non-uniform coarsening, as depicted in Fig. 10, in which some grains grow rather larger. It is no wonder that the grain distribution scatters to cover a comparatively large scope at $J_{\mathrm{e}}=120 \mathrm{~A}$, as indicated in Fig. 6 .

Another impact of joule heat on microstructure is that crystallized solids experience an annealing-like treatment. During the annealing process, individual atomic migration

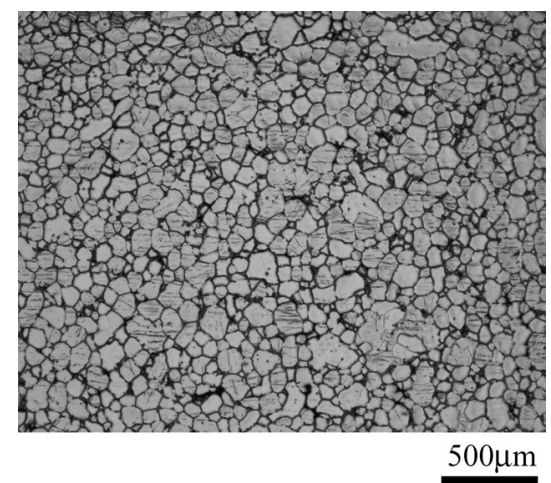

Fig. 10. The microstructure of the AZ31 alloy solidified at high electric current of $J_{\mathrm{e}}=120 \mathrm{~A}$ when $f=500 \mathrm{~Hz}$ and $B_{0}=10 \mathrm{~T}$, exhibiting a non-uniform feature with some abnormally coarsened grains.

is rather rapid and thus making the deformation twin lamellae frequently contact and some of them eventually disappear. ${ }^{30)}$ One good example to verify this concept is that few twins can be found in completely crystallized AZ31 alloys after rolling whereas a great number of deformation twins can be identified in the alloy after cold rolling. ${ }^{33)}$ This is why that one can find a fewer twins in the alloy solidified at $J_{\mathrm{e}}=120 \mathrm{~A}$. Here it becomes clear that the low number fraction of twinned grains at low and high $J_{\mathrm{e}}$ should be ascribed to different origins in that electromagnetic force may be insufficient to deform more grains to yield twins in the former case while in the latter case, twinned planes may annihilate because of a prolonged cooling process and thus resulting in a decreased number fraction of twins.

\section{Conclusions}

We have solidified the AZ31 alloy in a superconducting magnet with magnetic flux density up to $10 \mathrm{~T}$ when an alternating electric current passes through the alloy, under which EMV process can be produced. The microstructure formation of the alloy solidified under various vibration conditions was investigated systematically. The main conclusions can be drawn as follows:

(1) The electrical resistivity of liquid is about as high as twice that solid in the mushy zone at high temperatures, yielding the electric current in solid about twice that in liquid. Therefore, the Lorentz force to which the solid is subjected is about twice that liquid, resulting in a much higher acceleration and velocity and thus a larger displacement for the solid than the liquid. Consequently, an uncoupled movement between the solid and liquid occurs and the vibration even within one vibration cycle is not synchronous. Three different aspects can be influenced regarding the solidification of a dendrite due to this uncoupled movement; (i) relative motion takes place to yield melt flow that can promote the growing dendrite to segment into fine particles; (ii) the melt flow may rotate or carry fragmented particles to leave their original positions and thus breaking the primary dendritic skeleton and making texture random in orientation distribution; and (iii) the relative movement can induce interactive shear force that may deform the primary solid to generate deformation twins in the alloy. From the viewpoint of solute redistribution, the relative movement between the 
solid and liquid makes it difficult to establish a steady state diffusion layer for the solute rejected by the growing crystals and thus there are probably no constitutionally undercooled regions ahead of the solid/liquid interface and thus a dendrite and/or its higher branches cannot be formed.

(2) Regarding to the frequency-dependent microstructure development, it is revealed that refinement becomes the most effective when the relative displacement covered by the leading solid is as large as the thickness of solute redistribution layer.

(3) In terms of the influence of magnetic flux density and electric current on microstructure formation, one can readily tell that both $J_{\mathrm{e}}$ and $B_{0}$ have a linear relation with Lorentz force, indicating that the larger the $J_{\mathrm{e}}$ and $B_{0}$, the higher the relative velocity and relative displacement that the solid leads to the liquid and hence the more pronounced the refining effect will be induced. Consequently, fine structures will be yielded at large $J_{\mathrm{e}}$ and $B_{0}$.

(4) Joule heat released by electric current becomes striking at high levels of electric current, i.e., $J_{\mathrm{e}}=90$ and $J_{\mathrm{e}}=120 \mathrm{~A}$, making the structure coarsening in the semisolid state at an extended period during slow cooling. Meanwhile, the prolonged cooling process at high temperatures at the large current of $J_{\mathrm{e}}=120 \mathrm{~A}$ enables the annihilation of deformation twins.

\section{Acknowledgements}

The authors are grateful to Mr. H. Matsubara and Mr. D. Kamikihara for their technical assistance in ingot preparation. Thanks are also due to Dr. N. Saito for his kind help in EBSD analysis.

\section{REFERENCES}

1) Ch. Vives: Metall. Mater. Trans. B., 27B (1996), 445.

2) Ch. Vives: Metall. Mater. Trans. B., 27B (1996), 457.

3) Ch. Vives: Metall. Mater. Trans. B., 20B (1989), 623.

4) For a review see Ch. Vives: JOM-e, 50 (1998). http://www. tms.org/pubs/journals/JOM/9802/Vives/Vives-9802.html. (accessed August 20, 2006)

5) A. Radjai, K. Miwa and T. Nishino: Metall. Mater. Trans. A., 29A
(1998), 1477.

6) A. Radjai and K. Miwa: Metall. Mater. Trans. A., 31A (2000), 755.

7) A. Radjai and K. Miwa: Metall. Mater. Trans. A., 33A (2002), 3025.

8) Y. Mizutani, S. Kawai, K. Miwa, K. Yasue, T. Tamura and Y. Sakaguchi: Mater. Trans., 45 (2004), 1939.

9) Y. Mizutani, Y. Ohura, K. Miwa, K. Yasue, T. Tamura and Y. Sakaguchi: Mater. Trans., 45 (2004), 1944.

10) Y. Mizutani, J. Kawata, K. Miwa, K. Yasue, T. Tamura and Y. Sakaguchi: J. Mater. Res., 19 (2004), 2997.

11) Y. Mizutani, T. Tamura and K. Miwa: Mater. Sci. Eng. A., 413-414 (2005), 205.

12) Y. Mizutani, K. Miwa, T. Tamura, Y. Nakai and Y. Otsuka: Mater. Trans., 47 (2006), 1793.

13) T. Tamura, K. Amiya, R. S. Rachmat, Y. Mizutani, and K. Miwa: Nature Mater, 4 (2005), 289.

14) J. Dong, J. Cui, X. Zeng and W. Ding: Mater. Trans., 46 (2005), 94.

15) J. Dong, J. Cui, F. Yu, C. Ban and Z. Zhao: Metall. Mater. Trans. A., 35A (2004), 2487.

16) Y. Wang, X. Zeng, W. Ding, A. A. Luo and A. K. Sachdev: Metall. Mater. Trans. A., 38A (2007), 1358.

17) D. H. StJohn, M. Qian, M. A. Easton, P. Cao and Z. Hildebrand: Metall. Mater. Trans. A., 36A (2005), 1669.

18) E. A. Hidemann: J. Acoust. Soc. Am., 26 (1954), 831.

19) O. V. Abramov: Ultrasonics, 25 (1987), 73.

20) M. M. Avedesian and H. Baker: ASM Specialty Handbook Magnesium and Magnesium Alloys, The Materials International Society, Materials Park, OH, (1999), 9; 15.

21) J. R. Davis: ASM Specialty Handbook Aluminum and Aluminum Alloys, The Materials International Society, Materials Park, OH, (1993), 641.

22) J. R. Davis: ASM Specialty Handbook Copper and Copper Alloys, The Materials International Society, Materials Park, OH, (2001), 449.

23) M. C. Flemings: Metall. Trans. A., 22A (1991), 957.

24) A. Hellawell, S. Liu and S. Z. Lu: JOM, 49 (1997), March, 18.

25) M. Li, T. Ishikawa, K. Nagashio, K. Kuribayashi and S. Yoda: Acta Mater, 54 (2006), 3791.

26) D. J. Dingley and V. Randle: J. Mater. Sci., 27 (1992), 4545.

27) R. E. Reed-Hill and W. D. Robertson: Acta Metall., 5 (1957), 717.

28) J. Koike: Metall. Mater. Trans. A., 36A (2005), 1689.

29) Y. N. Wang and J. C. Huang: Acta Mater, 55 (2007), 897.

30) J. W. Christian and S. Mahajan: Progress Mater. Sci., 39 (1995), 1.

31) M. Li, T. Tamura and K. Miwa: unpublished results.

32) M. Li, T. Tamura and K. Miwa: Acta Mater, 55 (2007), 4635.

33) M. R. Barnett, Z. Keshavarz and M. D. Nave: Metall. Mater. Trans. A., 36A (2005), 1697. 